\title{
Recent Run II QCD Results from DØ
}

(To be published in the Proceedings of the $15^{\text {th }}$ Topical Conference on Hadron Collider Physics, HCP 2004, Michigan State University, East Lansing, MI, June 14-18, 2004)

\author{
Markus Wobisch
}

for the $\mathrm{D} \emptyset$ Collaboration

Fermilab, P.O. Box 500, Batavia, Illinois 60510

\begin{abstract}
We present recent QCD results from the DØ experiment at the Fermilab Tevatron Collider in $p \bar{p}$ collisions at $\sqrt{s}=1.96 \mathrm{TeV}$. Results are presented for the inclusive jet and dijet cross sections, a measurement of dijet azimuthal decorrelations, studies of elastic scattering, and a search for diffractively produced $Z$ bosons.
\end{abstract}

\section{INTRODUCTION}

The center-of-mass energy for proton-antiproton $(p \bar{p})$ collisions was increased from $\sqrt{s}=1.8 \mathrm{TeV}$ to $1.96 \mathrm{TeV}$ in Run II of the Tevatron Collider at Fermi National Accelerator Laboratory. This article presents QCD results measured with the D $\varnothing$ detector [1], based on data sets corresponding to integrated luminosities of $\approx 150 \mathrm{pb}^{-1}$. These are measurements of the inclusive jet and the dijet cross section, studies of azimuthal decorrelations in dijet production, a search for diffractively produced $Z$ bosons, and a measurement of the $d N / d|t|$ distribution in elastic $p \bar{p}$ scattering.

\section{JET PRODUCTION}

The production rates of particle jets with large transverse momentum $\left(p_{T}\right)$ provide sensitive tests for the predictions of perturbative QCD (pQCD) and give information on the non-perturbative structure of the proton as parameterized in the parton density functions (PDFs). The increased center-of-mass energy of $\sqrt{s}=1.96 \mathrm{TeV}$ in Run II results in an increased jet cross section, as compared to $\sqrt{s}=1.8 \mathrm{TeV}$ in Run I. Fig. 1 shows the ratio of the $\mathrm{pQCD}$ predictions for the inclusive jet cross section for both center-of-mass energies. At $p_{T}$ above $400 \mathrm{GeV}$ the cross section increases by more than a factor of two. At high integrated luminosity this increases the accessible $p_{T}$ range and extends the discovery potential for new physics phenomena. The increased cross section at high $p_{T}$ will also allow to test the dynamics of the strong interaction in unprecedented regions and constrain the PDFs at highest momentum fractions $x$. The sensitivity of the jet cross section to the PDFs can be seen from the decomposition of the total jet cross section into partonic subprocesses. This is shown in Fig. 1 (right) for the inclusive jet cross section as a function of $p_{T}$. Up to $p_{T}=200 \mathrm{GeV}$ (where the PDFs are probed at $x \approx 0.4$ ), the largest fraction of the jet cross section comes from gluon-induced subprocesses. There are still significant contributions, even at high $p_{T}$, from quark-gluon scattering, giving direct sensitivity to the gluon density in the proton even at highest $x$. 

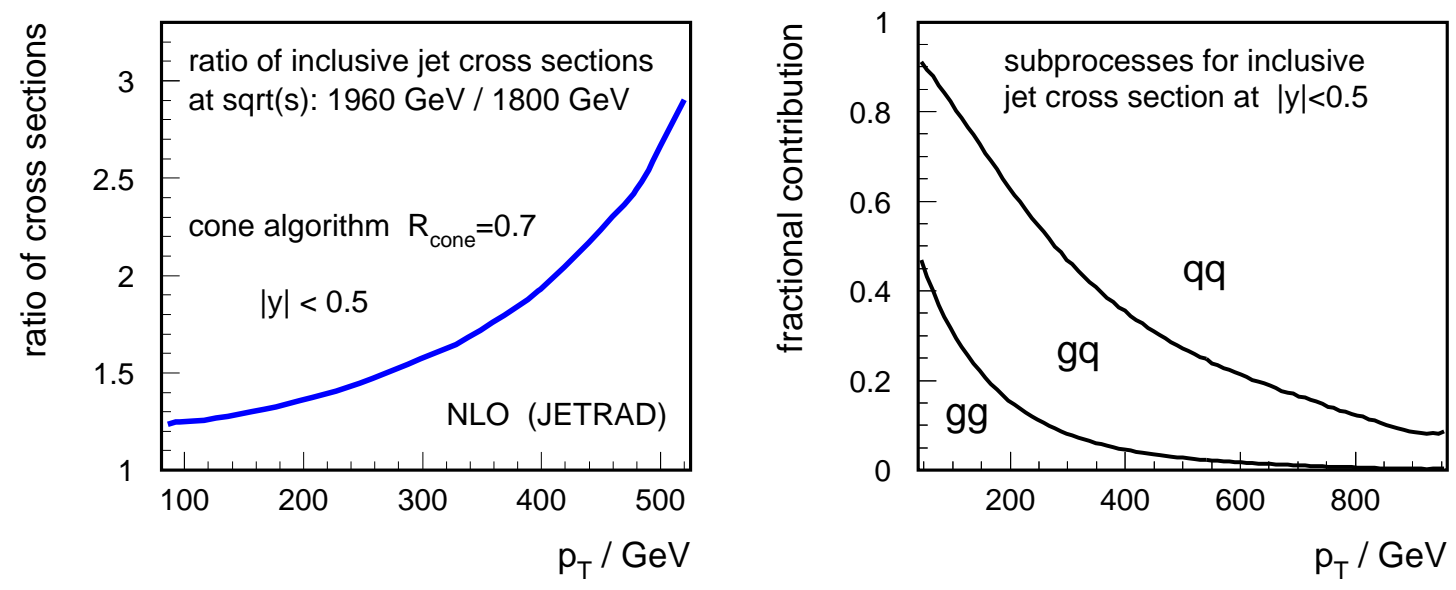

FIGURE 1. The ratio for the NLO pQCD predictions for the central inclusive jet cross section at different center-of-mass energies as a function of jet $p_{T}$ (left). The decomposition of the central inclusive jet cross section into different partonic subprocesses as a function of jet $p_{T}$ (right).

A major difference between the Tevatron jet measurements in Run I and Run II is the jet definition: the cone algorithm used in Run I was not infrared safe and reliable pQCD predictions were not possible [2]. The "Run II cone algorithm" [3] is still an iterative seed-based cone algorithm, but it uses midpoints between pairs of proto-jets as additional seeds which makes the procedure infrared safe [2]. A further difference between both jet algorithms is the chosen "recombination scheme". The Run I algorithm used the " $E_{T}$-scheme" (or "Snowmass convention" [4]) in which the transverse jet energy $E_{T}$ was defined as the scalar sum of the particle $E_{T}$ 's and the invariant jet mass was defined to be zero. Consequently, in Run I, the jet kinematics were described by $E_{T}$ and pseudorapidity, $\eta=-\ln (\tan (\theta / 2))$. In Run II particles are combined into jets using the " $E$-scheme" (addition of four-vectors). The $E$-scheme results in massive jets which are described by $p_{T}$ and rapidity, $y=\frac{1}{2} \ln \left(\left(E+p_{z}\right) /\left(E-p_{z}\right)\right)$. The $E$-scheme has the advantage that the kinematic boundary $p_{T}^{\max }=\sqrt{s} / 2$ is stable, independent of the number of particles in a jet (which was not the case in the $E_{T}$-scheme). This is desirable for resummed calculations [5].

All jet measurements presented here are using a data sample corresponding to an integrated luminosity of $\approx 150 \mathrm{pb}^{-1}$. The cone radius (in $y$ and $\phi$ space) was chosen to be $R_{\text {cone }}=0.7$. Jet detection was based on a compensating, finely segmented, liquidargon and uranium calorimeter that provided nearly full solid-angle coverage. Events were acquired using multiple-stage inclusive-jet triggers. Analysis regions were defined based on the jet with largest $p_{T}$ in an event $\left(p_{T}^{\max }\right)$ with the requirement that the trigger efficiency be at least $99 \%$. The position of the $p \bar{p}$ interaction was reconstructed using a tracking system consisting of silicon microstrip detectors and scintillating fibers located within a $2 \mathrm{~T}$ solenoidal magnet. The $p_{T}$ of each jet was corrected for calorimeter showering effects, overlaps due to multiple interactions and event pile-up, calorimeter noise effects, and the energy response of the calorimeter. The data were corrected for selection efficiencies and for migrations due to resolution. The largest experimental uncertainty is due to the jet energy calibration. 

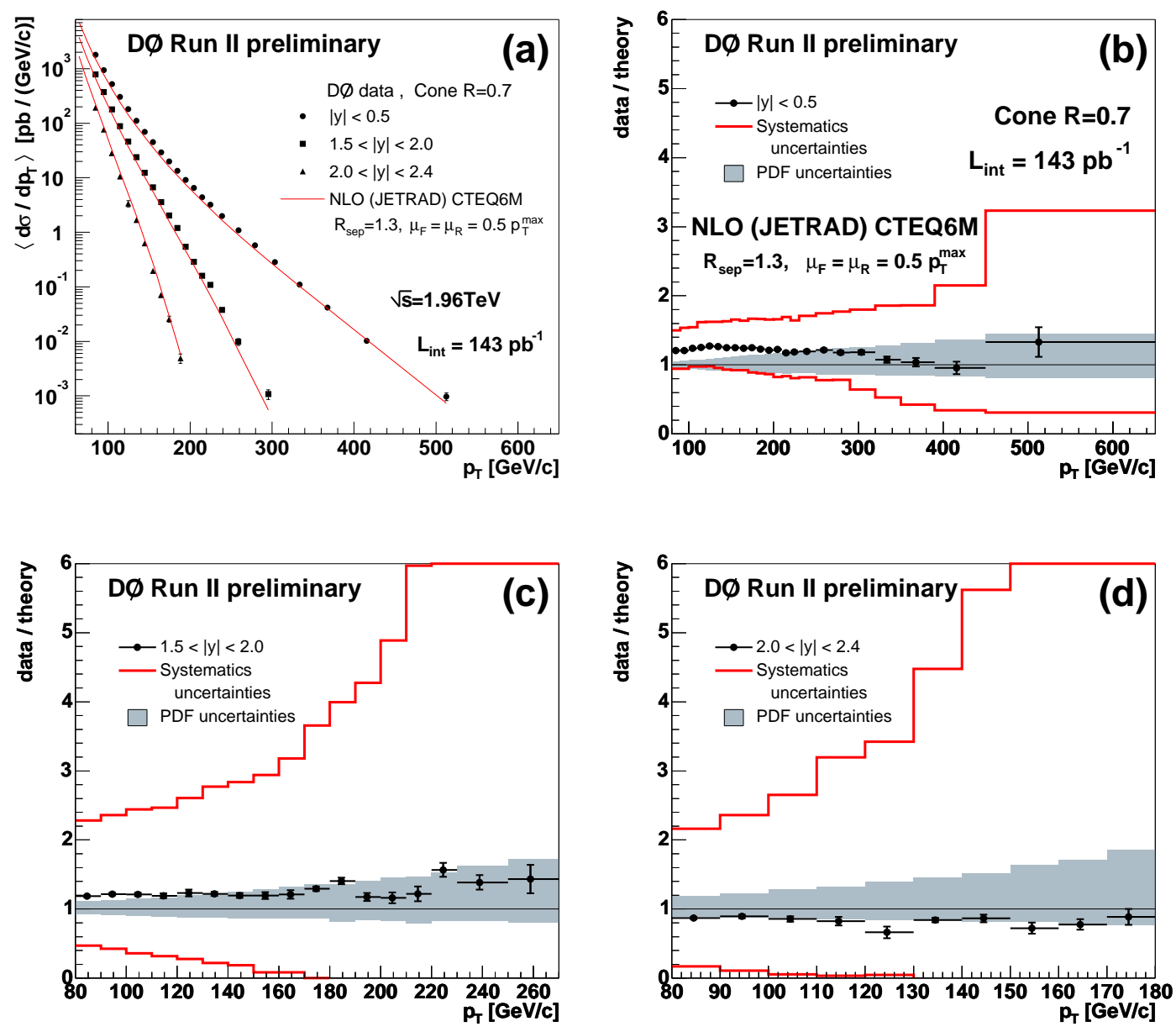

FIGURE 2. The inclusive jet cross section as a function of $p_{T}$ in different rapidity regions (a). Only statistical errors are shown. Figures (b), (c), (d) show the ratios of data and the NLO pQCD predictions. The error bars indicate the statistical errors and the total experimental uncertainty is displayed by the lines. Theoretical uncertainties due to the PDFs are shown as the shaded bands.

\section{Inclusive Jet and Dijet Jet Cross Sections}

The inclusive jet cross section is measured as a function of $p_{T}$ and $y$. Fig. 2 (a) shows the $p_{T}$ dependence of the inclusive jet cross section in three rapidity regions in the range $0<|y|<2.4$ (with statistical errors only). The cross section is falling by more than six orders of magnitude between $p_{T}=100 \mathrm{GeV}$ and $p_{T}=500 \mathrm{GeV}$. At forward rapidities the $p_{T}$ dependence is much stronger. Next-to-leading order (NLO) pQCD predictions are overlaid on the data. The NLO calculations [6] were computed for renormalization and factorization scales $\mu_{r}=\mu_{f}=0.5 p_{T}^{\max }$ using the CTEQ6M [7] PDFs and $\alpha_{s}\left(M_{Z}\right)=0.118$. The maximum distance of particles within a jet was limited to $\Delta R<R_{\text {sep }} \cdot R_{\text {cone }}$ with $R_{\text {sep }}=1.3$ [8]. The ratio of data over theory is shown in Figs. 2 (b), (c), (d). Uncertainties in the NLO calculations due to the PDFs are indicated by the grey bands; the experimental uncertainties are displayed as lines. The latter increase with $p_{T}$, especially at large rapidities. Theory has good agreement with data given the large 

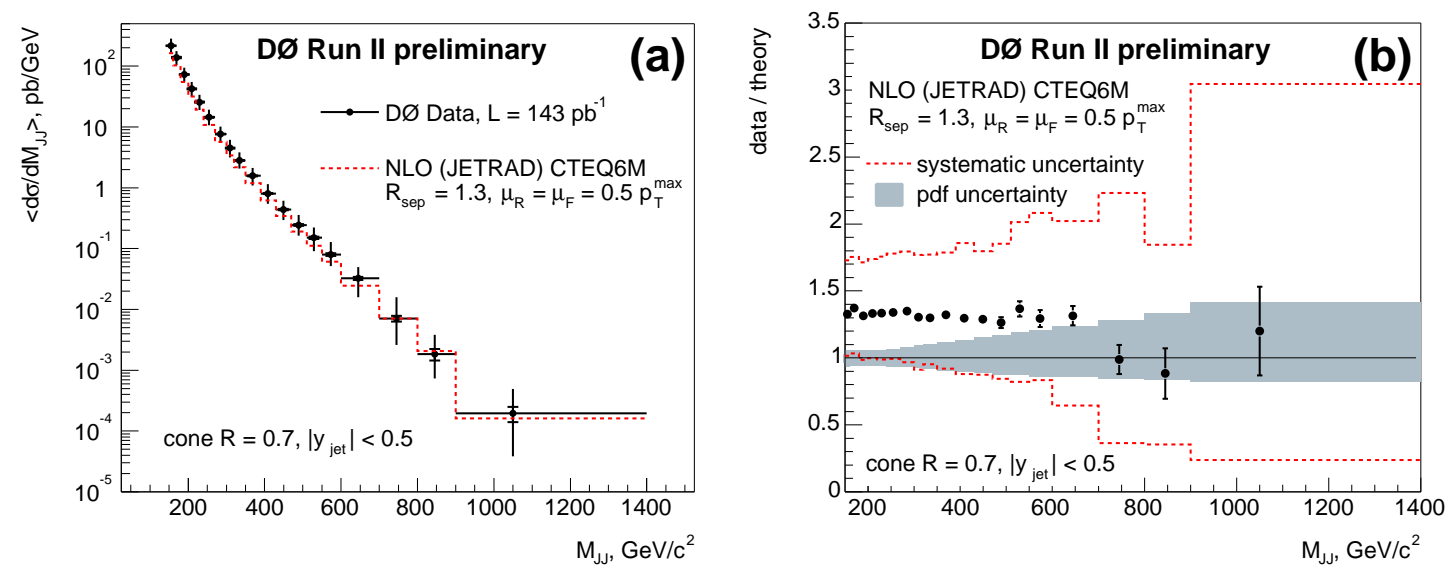

FIGURE 3. The dijet cross section as a function of $M_{\mathrm{jj}}$ at central rapidities. In Fig. (a) the data are shown with the total experimental uncertainties. The statistical uncertainties are displayed by the inner error bars. Fig. (b) shows the ratio of data and the NLO pQCD predictions. The error bars indicate the statistical errors and the total experimental uncertainty is displayed by the lines. Theoretical uncertainties due to the PDFs are shown as the shaded bands.

uncertainties. The dijet cross section at central rapidities $(|y|<0.5)$ is shown in Fig. 3 (a) as a function of the invariant dijet mass $M_{\mathrm{jj}}$ in the range $150<M_{\mathrm{jj}}<1400 \mathrm{GeV}$. The ratio of data and theory is displayed in Fig. 3 (b). Within the large experimental uncertainties theory gives a good description of the data.

It is clear that the current jet cross section measurements with their large uncertainties can not constrain the PDFs. Improvements in the jet energy calibration and a reduction of the corresponding uncertainty are still needed. It has to be noted that at the time this article is written significant improvements have already been achieved and further work is in progress.

\section{Dijet Azimuthal Decorrelations}

As long as the uncertainties of the jet energy calibration are still large it is convenient to investigate observables which have a smaller dependence on the jet energy calibration but which are still sensitive to QCD effects. The decorrelation of the azimuthal angles of the two leading $p_{T}$ jets, $\Delta \phi_{\text {dijet }}=\left|\phi_{\text {jet1 }}-\phi_{\text {jet } 2}\right|$, is such an observable. In lowest order pQCD both jets in a dijet event have equal $p_{T}$ and correlated azimuthal angles $\Delta \phi_{\text {dijet }}=\pi$. Additional soft radiation causes small azimuthal decorrelations, whereas $\Delta \phi_{\text {dijet }}$ significantly lower than $\pi$ is evidence of additional hard radiation with high $p_{T}$. Exclusive three-jet production populates $2 \pi / 3<\Delta \phi_{\text {dijet }}<\pi$ while smaller values of $\Delta \phi$ dijet require additional radiation such as a fourth jet in an event. Distributions in $\Delta \phi_{\text {dijet }}$ provide an ideal testing ground for higher-order pQCD predictions without requiring the reconstruction of additional jets and offer a way to examine the transition between soft and hard QCD processes based on a single observable.

The observable is defined as the differential dijet cross section in $\Delta \phi_{\text {dijet }}$, normalized by the inclusive dijet cross section integrated over the same phase space 

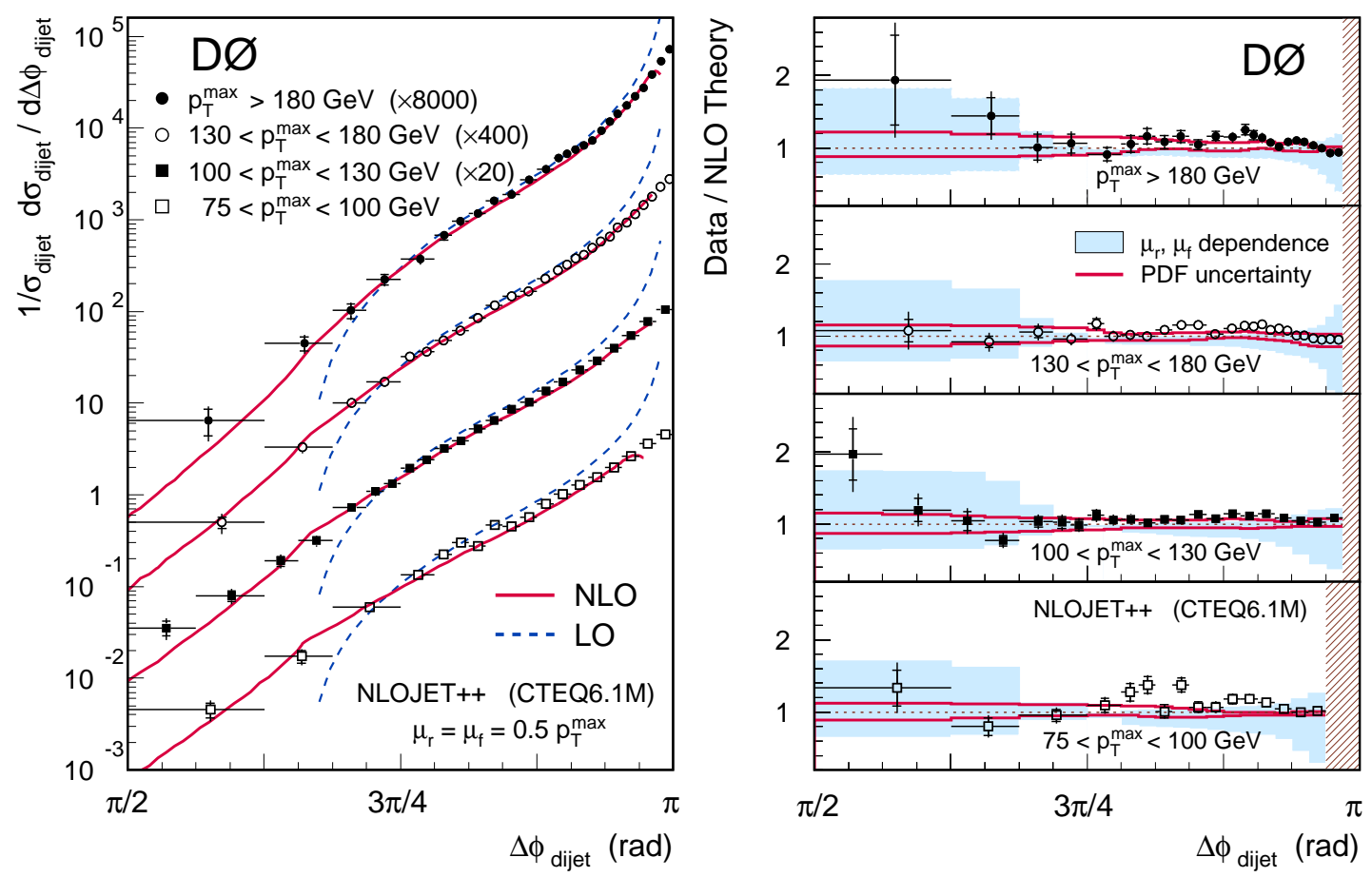

FIGURE 4. Left: The $\Delta \phi_{\text {dijet }}$ distributions in four regions of $p_{T}^{\max }$. The solid (dashed) lines show the NLO (LO) pQCD predictions. Right: Ratios of data to the NLO pQCD calculation for different regions of $p_{T}^{\max }$. Theoretical uncertainties due to variation of $\mu_{r}$ and $\mu_{f}$ are shown as the shaded regions; the uncertainty due to the PDFs is indicated by the solid lines. The points at large $\Delta \phi_{\text {dijet }}$ are excluded because the calculation has non-physical behavior near the divergence at $\pi$.

$\left(1 / \sigma_{\text {dijet }}\right)\left(d \sigma_{\text {dijet }} / d \Delta \phi_{\text {dijet }}\right)$. The corrected data [9] are presented in Fig. 4 (left) as a function of $\Delta \phi_{\text {dijet }}$ in four ranges of $p_{T}^{\max }$. The inner error bars represent the statistical uncertainties and the outer error bars correspond to the total experimental uncertainties. The spectra are strongly peaked at $\Delta \phi_{\text {dijet }} \approx \pi$ and the peaks are narrower at larger values of $p_{T}^{\max }$. The data in Fig. 4 (left) are compared to the pQCD predictions in leading order (LO) and NLO [10] for $\mu_{r}=\mu_{f}=0.5 p_{T}^{\max }$ using CTEQ6.1M [7] PDFs. The (N)LO predictions are computed as the ratio of the $(\mathrm{N}) \mathrm{LO}$ predictions for $2 \rightarrow 3$ processes and $2 \rightarrow 2$ processes. Due to kinematic constraints, the $\mathrm{LO}$ calculation is restricted to $\Delta \phi_{\text {dijet }}>2 \pi / 3$. At $\Delta \phi_{\text {dijet }}=\pi$ where the third jet becomes soft the LO predictions diverge. NLO pQCD provides a good description of the data. This is emphasized in Fig. 4 (right) which displays the ratio of data and NLO. There is good agreement within a small offset of 5-10\% relative to unity. Theoretical uncertainties due to the PDFs [7] are estimated to be below $20 \%$. Also shown are the effects of $\mu_{r}$ and $\mu_{f}$ variations in the range $0.25 p_{T}^{\max }<\mu_{r, f}<p_{T}^{\max }$. The large scale dependence for $\Delta \phi_{\text {dijet }}<2 \pi / 3$ occurs because the NLO calculation only receives contributions from tree-level four-parton final states in this regime. NLO pQCD fails to describe the data in the region $\Delta \phi_{\text {dijet }} \approx \pi$ which is dominated by soft processes and therefore can not be described in fixed-order perturbation theory. pQCD results at large $\Delta \phi_{\text {dijet }}$ in Figs. 4 were excluded because the calculations have non-physical behavior near the divergence at $\pi$ and were not stable. 

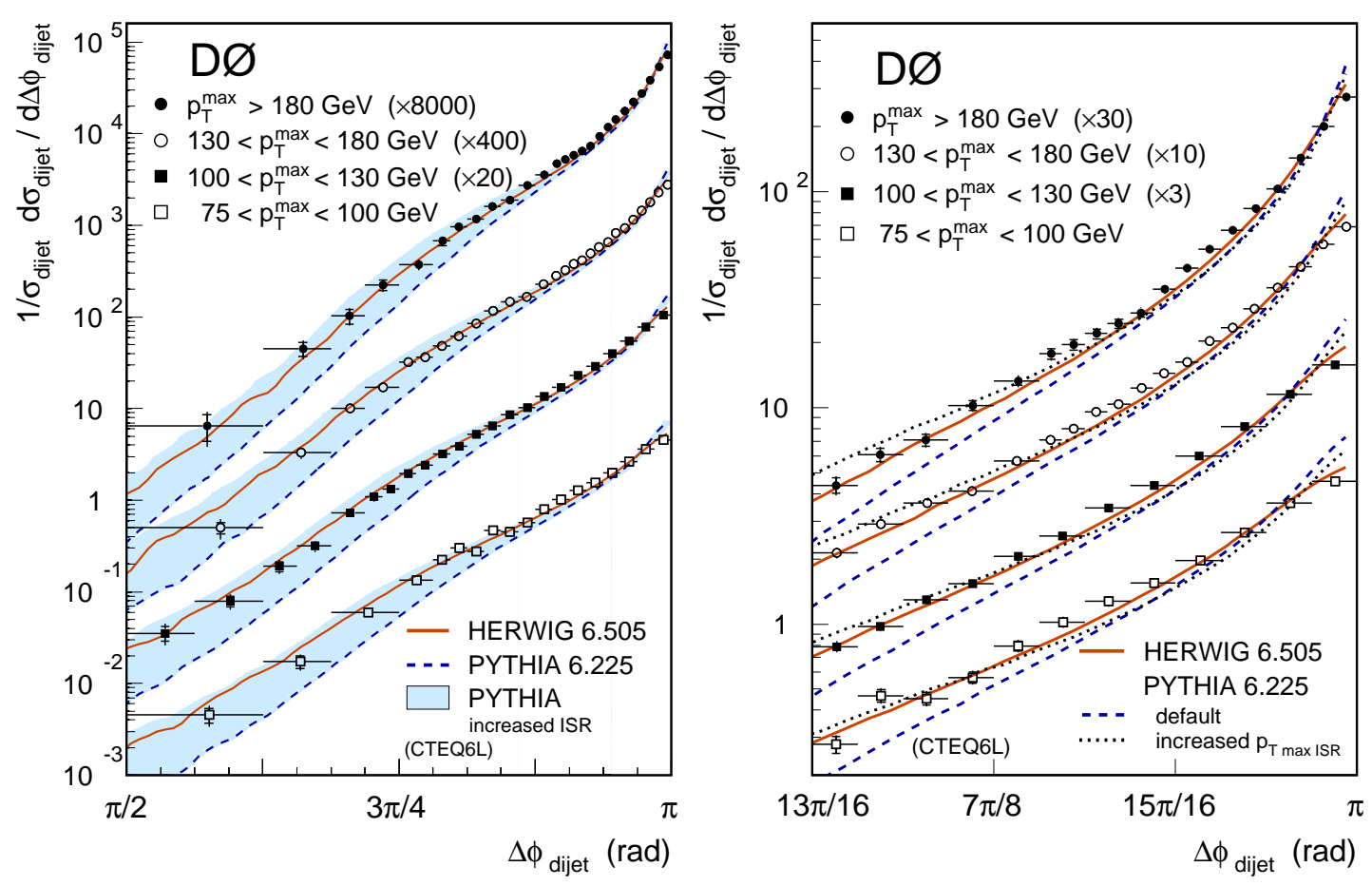

FIGURE 5. The $\Delta \phi_{\text {dijet }}$ distributions in different $p_{T}^{\max }$ ranges. Results from HERWIG and PYTHIA are overlaid on the data. The left Figure displays the full $\Delta \phi_{\text {dijet }}$ range and the right Figure emphasizes the region close to $\pi$.

Monte Carlo event generators, such as HERWIG and PYTHIA, use $2 \rightarrow 2$ LO pQCD matrix elements with phenomenological parton-shower models to simulate higher order QCD effects. HERWIG and PYTHIA results for default parameters, CTEQ6L [7] PDFs, and $\alpha_{s}\left(M_{Z}\right)=0.118$, are compared to the data in Fig. 5 over the whole $\Delta \phi_{\text {dijet }}$ range (left) and in greater detail in the region $\Delta \phi_{\text {dijet }}>13 \pi / 16$ (right). The default version of HERWIG gives a good description of the data over the whole $\Delta \phi_{\text {dijet }}$ range in all $p_{T}^{\max }$ regions. It is slightly below the data around $\Delta \phi_{\text {dijet }} \approx 7 \pi / 8$ and slightly narrower peaked at $\pi$. The default version of PYTHIA does not describe the data. The distribution is too narrowly peaked at $\Delta \phi_{\text {dijet }} \approx \pi$ and lies significantly below the data over most of the $\Delta \phi_{\text {dijet }}$ range in all $p_{T}^{\max }$ regions.

To investigate the possibilities of tuning PYTHIA we focus on the impact of the initialstate radiation (ISR) parton shower and the parameters by which the ISR shower can be adjusted. The maximum allowed $p_{T}$ in the ISR shower is controlled by the product of the hard scattering scale $\left(p_{T}^{2}\right)$ and the parameter PARP (67). Increasing this cut-off from the current default of 1.0 to the earlier default of 4.0 leads to significant changes of the PYTHIA prediction for $\Delta \phi_{\text {dijet }}$ (the shaded bands in Fig. 5, left, and the dotted line in Fig. 5, right). At low $\Delta \phi_{\text {dijet }}$ the description of the data becomes very good but at large $\Delta \phi$ dijet this parameter has not enough effect to bring PYTHIA close to the data. The best description of the low $\Delta \phi_{\text {dijet }}$ region is obtained for PARP ( 67) $=2.5$ (not shown).

Further ISR-related parameters are tested if they have impact on the $\Delta \phi_{\text {dijet }}$ distribution. These are the scale factor, $x_{\mu}$, for the renormalization scale for $\alpha_{s}$ in the ISR 

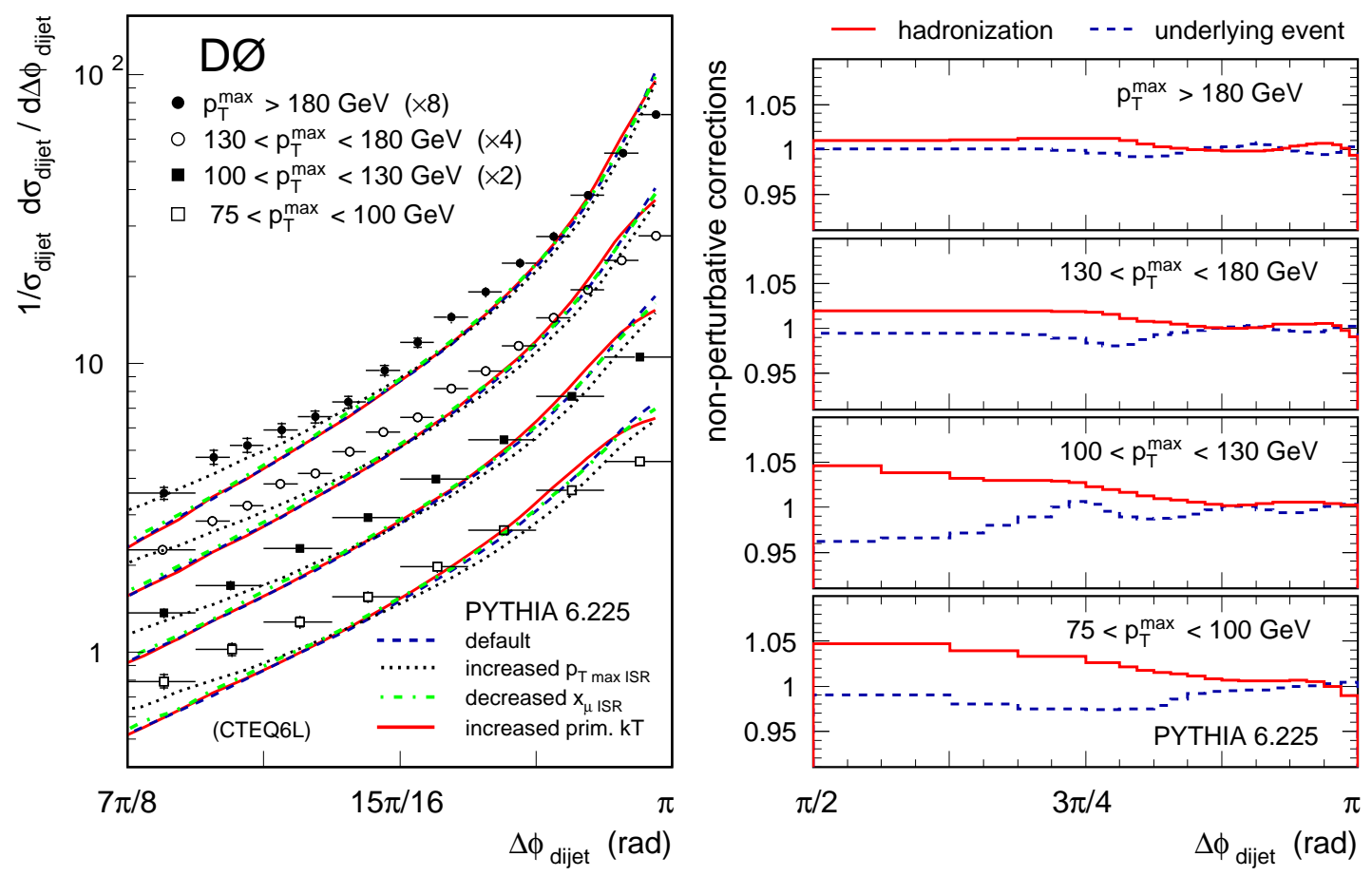

FIGURE 6. Left: The $\Delta \phi_{\text {dijet }}$ distributions in different $p_{T}^{\max }$ ranges. Results from PYTHIA are overlaid on the data for different parameter settings of the ISR shower. Right: Non-perturbative corrections for the $\Delta \phi_{\text {dijet }}$ distribution. Shown are the hadronization corrections (solid line) and the effects from underlying event as determined using PYTHIA.

shower, PARP (64), and the primordial $k_{T}$ of partons in the proton: the central value of the gaussian distribution, PARP (91), and the upper cut-off, PARP (93). We have lowered the factor for the renormalization scale to PARP $(64)=0.5 \quad(D=1.0)$ which increases the value of $\alpha_{s}$ according to the RGE. We have alternatively increased the primordial $k_{T}$ from $1 \mathrm{GeV}$ to $4 \mathrm{GeV}$, PARP $(91)=4.0 \quad(\mathrm{D}=1.0)$, and the upper cut-off of the gaussian distribution from $5 \mathrm{GeV}$ to $8 \mathrm{GeV}$, PARP $(93)=8.0 \quad(D=5.0)$. These parameter variations have no effect on the region at low $\Delta \phi_{\text {dijet }}$ and the effects at large $\Delta \phi_{\text {dijet }}$ are shown in Fig. 6 (left). Both effects are very small: while the scale factor has almost zero influence (dashed-dotted line) there is some small change for the increased primordial $k_{T}$ (solid line) which manifests itself, however, only very close to the peak region and only at lower $p_{T}^{\max }$.

Non-perturbative corrections for the $\Delta \phi_{\text {dijet }}$ distribution are investigated in Fig. 6 (right) using PYTHIA. Hadronization corrections are defined as the ratio of the observable, defined on the level of stable particles and on the level of partons. The underlying event correction is defined as the ratio of the predictions including underlying event and with the underlying event switched off. Both corrections are below 5\%, decreasing with $p_{T}^{\max }$. The smallness of these corrections allows to directly compare the measurement to purely perturbative QCD predictions and it also allows to interpret the discrepancies of the Monte Carlo event generators in terms of perturbative processes. 


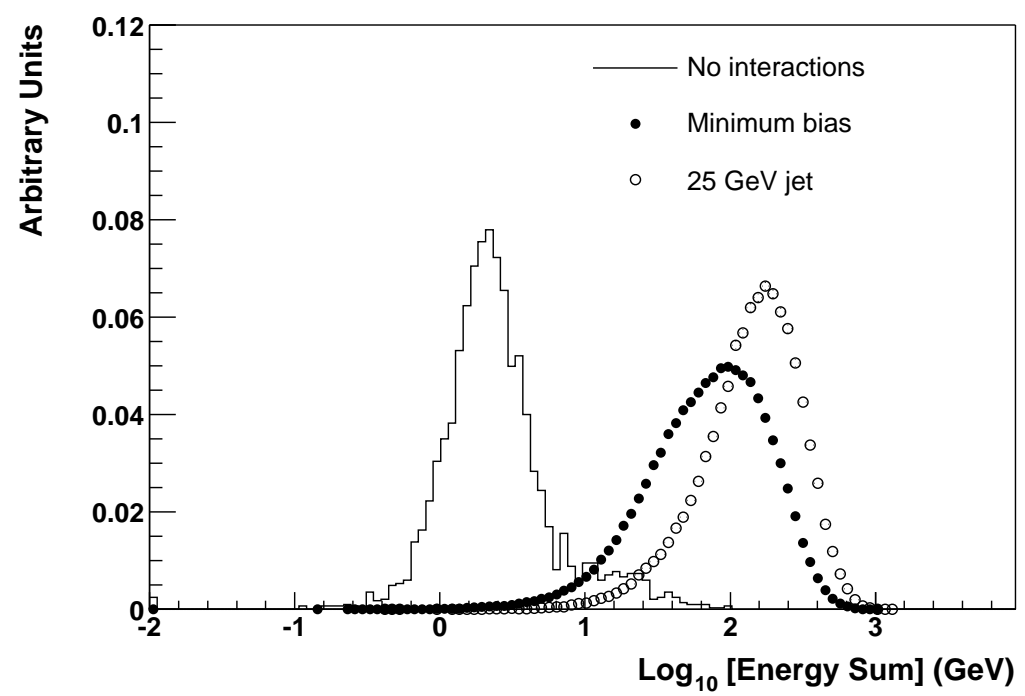

FIGURE 7. The logarithm of the reconstructed energy in the direction of the outgoing antiproton in the range $-5.3<\eta<-2.6$. Compared are the distributions for events with no visible interactions (line) and events in which the proton and the anti-proton dissociate (full and open markers). The areas are normalised to unity. Rapidity gap candidates are selected by applying a cut at $10 \mathrm{GeV}$.

\section{ELASTIC AND DIFFRACTIVE SCATTERING}

So far we have discussed inelastic processes with large transverse momentum. However, only a small contribution to the total $p \bar{p}$ cross section comes from such hard inelastic processes. About $40 \%$ of the total $p \bar{p}$ cross section is elastic scattering and diffraction. In elastic scattering both protons are scattered with no momentum loss under a small angle and emerge intact. No further particles are produced. In single diffraction only one of the protons remains intact and the other proton dissociates. While additional particles can be produced, there is often a large rapidity interval towards the intact proton in which no particles are emitted (rapidity gap). Both elastic scattering and diffractive processes can be identified experimentally by either measuring the scattered protons or by reconstructing the rapidity gap as an angular region with very little energy in the calorimeter. In the following sections we present a search for diffractively produced $Z$ bosons in the muon decay channel and a preliminary spectrum of events in elastic scattering as a function of the four-momentum transfer to the proton.

\section{Diffractive Z Boson Production}

DØ collected an event sample of about $21 \mathrm{pb}^{-1}$ during Run I suitable for diffractive physics studies. Nine diffractively produced $Z \rightarrow e^{+} e^{-}$candidates were observed in this data sample [13]. The current search for $Z$ bosons, produced in single diffraction, uses a dataset of $\approx 110 \mathrm{pb}^{-1}$. The analysis is based on the experimental reconstruction of a rapidity gap close to the direction of the final-state (anti-) proton and the reconstruction 

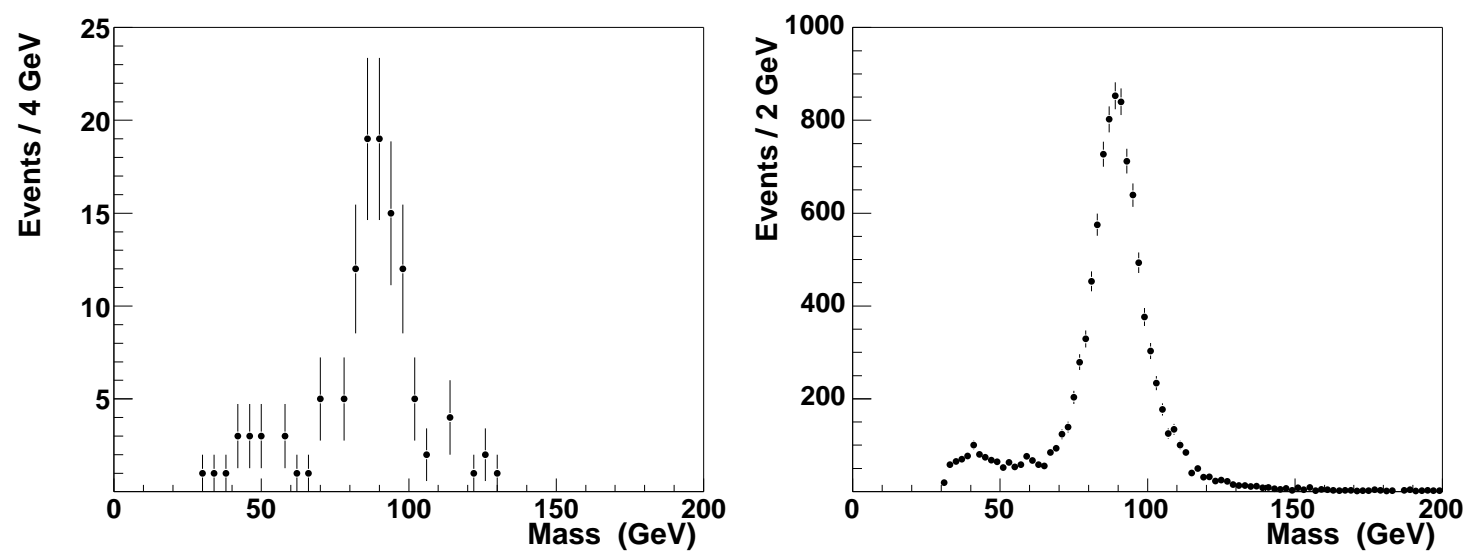

FIGURE 8. The invariant di-muon invariant mass distribution for $Z$ boson candidates with a single rapidity gap (left) and with no rapidity gap (right). For details on the rapidity gap definition, please see the text.

of the $Z$ via its decay into two muons with opposite charge. Both muons were required to have $p_{T}>15 \mathrm{GeV}$. One of the muons must be isolated in the calorimeter and in the central tracking detector. Muons from cosmic rays were suppressed by the requirement that their tracks stem from the vertex of the $p \bar{p}$ interaction and that their acolinearity $\left(\Phi=\Delta \phi_{\mu \mu}+\Delta \theta_{\mu \mu}-2 \pi\right)$ is larger than $0.05[14,15]$. The search of the rapidity gaps uses two scintillating detectors, one on each side of the interaction region, which cover the pseudorapidity range $2.7<|\eta|<4$.4. Calorimeter energies towards the directions of the outgoing protons (in the range $2.6<|\eta|<5.3$ ) are summed separately on each side with cell thresholds of $100 \mathrm{MeV}$ in the electromagnetic layers and $200 \mathrm{MeV}$ in the fine hadronic layer. The distribution of the logarithm of the energy sum is shown in Fig. 7 for bunch crossings with no visible interactions. Based on a random trigger these events are expected to have similar topologies as rapidity gap events with no activity in the direction of the outgoing antiproton. Also shown is the energy distribution for a sample of minimum bias events. A third event sample is defined by the requirement of (at least) one jet with $p_{T}>25 \mathrm{GeV}$. Events are excluded when the leading $p_{T}$ jet is in the region $|\eta|>2$.4. Both the minimum bias and the jet samples are dominated by non-diffractive events in which both protons dissociate. Events where the antiproton remains intact and events with antiproton dissociation can be separated by requiring the energy sum to be below $10 \mathrm{GeV}$. The final selection of single diffractive $Z$ boson candidates requires that on one side the scintillator is off and the energy sum is below $10 \mathrm{GeV}$ while on the other side the scintillator is on and the energy sum is larger than $10 \mathrm{GeV}$.

The invariant di-muon mass distribution is shown in Fig. 8 for $Z$ boson candidates in events with a rapidity gap (left) and in events that fail the rapidity gap selection (right). The latter are strong candidates for non-diffractively produced $Z$ bosons. Their mass distribution has a strong peak at the $Z$ mass and small background. The di-muon mass distribution for the candidates for diffractively produced $Z$ bosons has very similar shape. Additional studies on the purity and the background for the rapidity gap selection are, however, still needed for quantitative statements and to establish the evidence for a diffractive $Z \rightarrow \mu^{+} \mu^{-}$signal. 


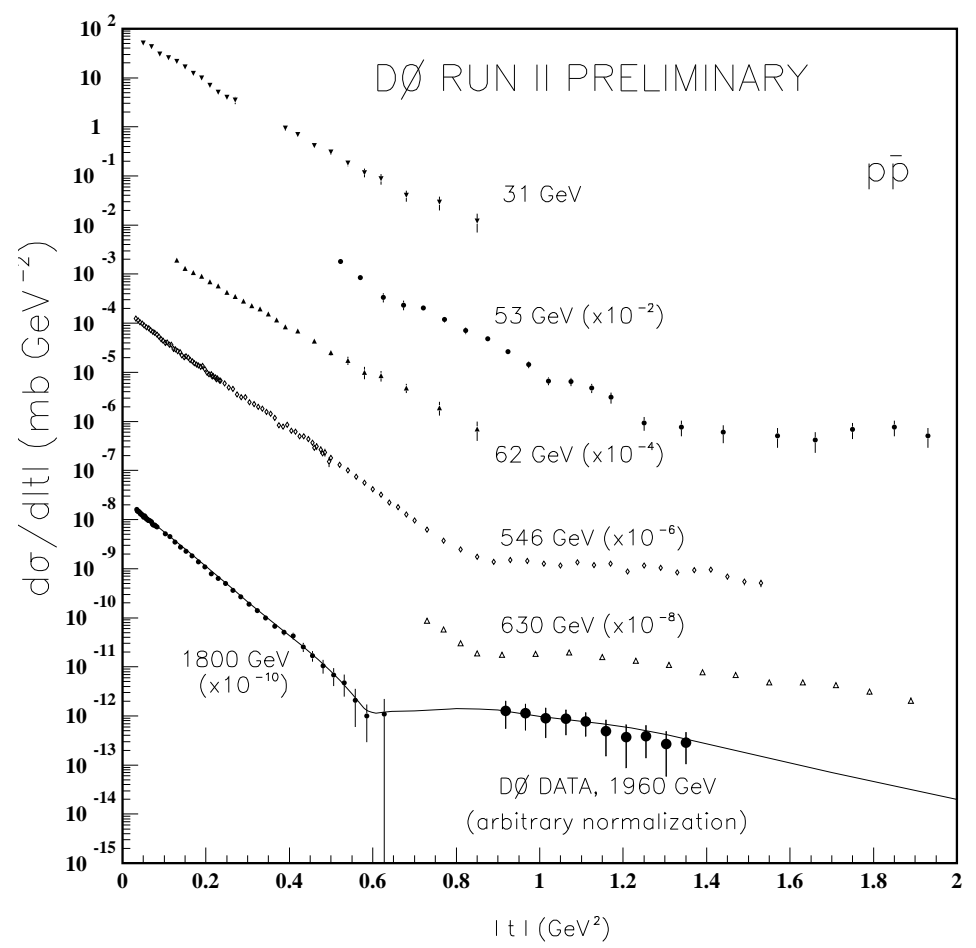

FIGURE 9. The differential elastic $p \bar{p}$ scattering cross section as a function of the four-momentum transfer squared $|t|$. Measurements from experiments at different center-of-mass energies are shown (datasets at different energies are scaled for visual separation). The preliminary D $\varnothing$ data are arbitrarily normalized for comparison of the slope with the E710 data at $\sqrt{s}=1.8 \mathrm{TeV}$. Theoretical predictions from Block et al. are overlaid on the data.

\section{Elastic Scattering}

In the DØ experiment an intact outgoing (anti-) proton can be detected in the Forward Proton Detector (FPD) [16], which consists of position detectors along the beam line in conjunction with accelerator magnets. In total, the FPD has nine spectrometers, each comprising two scintillating fiber tracking detectors that can be moved to within a few millimeters of the beam. The kinematics of the outgoing (anti-) proton is described by the four-momentum transfer squared, $t$ and the fractional longitudinal momentum loss $\xi$. Both variables $|t|$ and $\xi$ can be computed from the track four-vectors reconstructed in the FPD.

We have measured the $|t|$ distribution in elastic scattering using the FPD. Elastic events were triggered by two spectrometers which are above (below) the beam on the side of the outgoing antiprotons (protons). The spectrometer on the proton side was used to measure the track position. The trigger uses time-of-flight information, and vetoes events with hits in the scintillators covering $5.2<|\eta|<5.9$. The spectrometers can reconstruct protons in the kinematic range $0.8 \lesssim|t| \lesssim 4.0 \mathrm{GeV}^{2}$, depending on detector position and accelerator conditions. The preliminary results are presented in the range $0.92<|t|<1.34 \mathrm{GeV}^{2}$.

A Monte Carlo event generator was used to propagate the outgoing (anti-) protons in 
elastic scattering through the magnetic field of the Tevatron and to simulate the response of the FPD based on the expected detector resolution [17]. The geometric acceptance of the FPD was modeled by a similar event generator [18] as a function $|t|$. Remaining backgrounds from beam halo [19] and from single diffraction are subtracted separately.

The preliminary $d N / d|t|$ distribution for elastic $p \bar{p}$ scattering is shown in Fig. 9. Measurements of the differential cross section $d \sigma / d|t|$ at different center-of-mass energies from the ISR, UA4, and E710 experiments are also shown [20]. The normalization of the $\mathrm{D} \varnothing$ data is arbitrary, but it is visible that the shape of the $\mathrm{D} \varnothing$ data in the range $0.8<|t|<1.4 \mathrm{GeV}$ is very different from the shape of the E710 data measured at $\sqrt{s}=1.8 \mathrm{TeV}$ and at $|t|<0.65 \mathrm{GeV}$. A theoretical prediction by Block et al. [21] is compared to the data at highest center-of-mass energies. The model predicts a transition between the different $|t|$ regions and give a good description of the data over the whole $|t|$ range.

\section{REFERENCES}

1. V. Abazov et al. (DØ Collaboration), in preparation for submission to Nucl. Instrum. Methods Phys. Res. A.; T. LeCompte and H. T. Diehl, Ann. Rev. Nucl. Part. Sci. 50, 71 (2000); S. Abachi et al. (DØ Collaboration), Nucl. Instrum. Methods Phys. Res. A 338, 185 (1994).

2. M. H. Seymour, Nucl. Phys. B 513 (1998) 269.

3. G. C. Blazey et al., in Proceedings of the Workshop: "QCD and Weak Boson Physics in Run II", edited by U. Baur, R. K. Ellis, and D. Zeppenfeld, Batavia, Illinois (2000) p. 47. See Section 3.5.

4. J.E. Huth et al., in Proceedings of Research Directions For The Decade: Snowmass 1990, July 1990, edited by E.L. Berger (World Scientific, Singapore, 1992) p. 134.

5. S. Catani et al., Report of the QCD and Standard Model Working Group for the Workshop on Physics at TeV Colliders, Les Houches, France, 8-18 Jun 1999, p. 132.

6. W.T. Giele, E.W.N. Glover, and D.A. Kosower, Phys. Rev. Lett. 73 (1994) 2019.

7. J. Pumplin et al., JHEP 0207 (2002) 12; D. Stump et al., JHEP 0310 (2003) 046.

8. $\quad$ S.D. Ellis, Z. Kunszt and D.E. Soper, Phys. Rev. Lett. 69 (1992) 3615.

9. V. Abazov et al. (DØ Collaboration), submitted to Phys. Rev. Lett., hep-ex/ 0409040.

10. Z. Nagy, Phys. Rev. Lett. 88 (200 1220032); Z. Nagy, Phys. Rev. D 68 (2003) 094002.

11. T. Sjöstrand et al., Comp. Phys. Comm. 135 (2001) 238.

12. G. Marchesini et al., Comp. Phys. Comm. 67 (1992) 465; G. Corcella et al., JHEP 0101 (2001) 010.

13. V. Abazov et al. (DØ Collaboration), Phys. Lett. B 574 (2003) 169.

14. A. Lyon, these proceedings.

15. T. Edwards, in Proceedings of the 12th International Workshop on Deep Inelastic Scattering, Strbske Pleso, Slovakia, 14-18 Apr 2004.

16. A. Brandt et al. (DØ Collaboration), FERMILAB-PUB-97-377.

17. J. Molina and V. Oguri, Nov 2002. Available at: <http://alpha1.lafex.cbpf.br/ molina/elastic_mc/>

18. J. Barreto and J. Montanha, Jan 2003. Available at: $<$ http://www.ifi.unicamp.br/ montanha/fpd/sources/quadrupoles/pot_ver.for $>$

19. A. I. Drozhdin et al., FERMILAB-FN-0734.

20. A. Breakstone et al. (AMES-BOLOGNA-CERN-DORTMUND-HEIDELBERG-WARSAW Collaboration), Nucl. Phys. B 248 (1984) 253; A. Breakstone et al., Phys. Rev. Lett. 54 (1985) 2180; R. Battiston et al. (UA4 Collaboration), Phys. Lett. B 127 (1983) 472; M. Bozzo et al. (UA4 Collaboration), Phys. Lett. B 155 (1985) 197; D. Bernard et al. (UA4 Collaboration), Phys. Lett. B 171 (1986) 142; N. A. Amos et al. (E-710 Collaboration), Phys. Lett. B 247 (1990) 127.

21. M. Block et al., Phys. Rev. D 41 (1990) 978. 